\title{
ORTHOGONAL POLYNOMIALS WITH RATIO ASYMPTOTICS
}

\author{
VILMOS TOTIK
}

(Communicated by J. Marshall Ash)

\begin{abstract}
A general construction is given for measures for which the corresponding orthogonal polynomials have ratio asymptotics.
\end{abstract}

Let $\nu$ be a positive Borel measure on the unit circle $T$ and form the orthonormal polynomials $p_{n}(\nu, z)=\gamma_{n}(\nu) z^{n}+\cdots, n=0,1, \ldots$, with respect to $\nu$ :

$$
\int p_{n}(\nu, z) \overline{p_{m}(\nu, z)} d \nu(z)=\delta_{n m}
$$

We say that these polynomials have ratio asymptotic behavior if

$$
\lim _{n \rightarrow \infty} p_{n+1}(\nu, z) / p_{n}(\nu, z)=z
$$

uniformly on compact subsets of the exterior of the unit circle, and in this case we say shortly that $\nu$ is in the class $M$. If $\nu$ is given on the interval $[-1,1]$, then the corresponding class $M(0,1)$ is defined by the relation

$$
\lim _{n \rightarrow \infty} p_{n+1}(\nu, z) / p_{n}(\nu, z)=z+\sqrt{z^{2}-1}
$$

uniformly on compact subsets of $\mathbb{C} \backslash[-1,1]$. We mention, that the right-hand sides of (1) and (2) are the corresponding conformal mappings of the outer domains of the supports onto the exterior of the unit disk, and if a ratio asymptotic exists in the outer domains, then it must be of the form (1) or (2). There is a natural one-to-one correspondence between the measures in $M(0,1)$ and the even measures of $M$ that is established by the usual projection of the unit circle onto $[-1,1]$ [7].

The importance of the classes $M$ and $M(0,1)$ is explained by the connection between orthogonal polynomials, Padé approximation and continued fractions for Markov functions, and many investigations concerning the convergence properties of these quantities can be carried out (and have been carried out) for the case when the measure is in the class $M$ or $M(0,1)$. In fact, several recent investigations by A. A. Gonchar and E. A. Rahmanov indicate

Received by the editors August 7, 1990.

1980 Mathematics Subject Classification (1985 Revision). Primary 42C05; Secondary 39A11, 41 A99.

Key words and phrases. Orthogonal polynomials, ratio asymptotics, class $M$, singular measure.

The author's work was partially supported by the Hungarian National Science Foundation for Research, Grant No. 1157. 
that besides Szegö's condition, the condition $\nu \in M$ or $\nu \in M(0,1)$ seems to be the most natural condition for applications of orthogonal polynomials, see also [6].

The most general criterion for ensuring $\nu \in M$ is due to E. A. Rahmanov [7] who proved that $\nu^{\prime}>0$ almost everywhere on $T$ implies $\nu \in M(\nu \in$ $M(0,1))$. Here $\nu^{\prime}$ denotes the Radon-Nikodym derivative of the absolutely continuous part of $\nu$ with respect to linear Lebesgue measure on $T([-1,1])$. Several recent investigations have aimed at showing that $M$ is a considerably larger class than is suggested by Rahmanov's result. For example, several constructions were given in [1, 3-5] to show that $M$ contains discrete measures, and in [4] D. S. Lubinsky gave a general procedure that allowed him to show singular measures in $M$ without point masses. In connection with these results A. A. Gonchar recently asked if Rahmanov's condition is necessary for $\nu \in M$ if we assume that the measure $\nu$ has continuous density function. Below we show that this is not the case.

The aim of this note is to prove a result which, without hardly any computation, provides us with a flexible construction of measures in the class $M$ with certain prescribed properties. As special cases we can get the above-mentioned results concerning singular measures in $M$.

Theorem. Let $T$ be the unit circle, $m$ the normalized arc measure on $T, r>1$ a fixed number, and suppose that for each natural number $j$ there is given a sequence $\left\{\nu_{k}^{j}\right\}_{k=1}^{\infty}$ of Borel measures on $T$ which converges to $j^{-r} m$ in the weak*-topology. Then there exists a sequence $\left\{k_{j}\right\}$ such that the measure

$$
\nu=\sum_{j=1}^{\infty} \nu_{k_{j}}^{j}
$$

is in the class $M$.

As an immediate corollary we get the following result which is simpler in formulation: Let $r>1$. If $\left\{\nu_{k}\right\}_{k=1}^{\infty}$ is any sequence of measures on $T$ converging to the arc measure in the weak ${ }^{*}$-topology, then for some sequence $\left\{k_{j}\right\}$ the measure

$$
\nu=\sum_{j=1}^{\infty} j^{-r} \nu_{k_{j}}
$$

belongs to the class $M$.

Of course, both here and in the theorem the arc measure $m$ does not play a distinguished role. We have chosen it for convenience but it could be replaced by any other measure satisfying Szegö's condition.

Before giving the proof we mention a few possible choices for the sequences $\left\{\nu_{k}^{j}\right\}$.

(1) If each $\nu_{k}^{j}$ is a discrete measure then we get a discrete $\nu$ in $M$.

(2) Similarly, if each $\nu_{k}^{j}$ is a singular measure without mass point then we obtain a measure in $M$ with the same properties.

(3) If we choose the $\nu_{k}^{j}$ in such a way that it has continuous density $w_{k}^{j}$ with the properties $\left|w_{k}^{j}\right| \leq j^{-2}, m\left(\operatorname{supp}\left(w_{k}^{j}\right)\right)<\varepsilon(j+1)^{-2}$, where $\varepsilon$ is some given positive number, then we get a $\nu$ in $M$ that has continuous density $w$ and whose support has linear Lebesgue measure smaller than $\varepsilon$. 
(4) As a common generalization of (1) and (2) we get the following: if $\mu$ is any measure with support $T$ then there is a $\nu \in M$ that is absolutely continuous with respect to $\mu$ (we also mention that the support of $\mu$ must be equal to $T$ if we want this conclusion). In fact, by choosing $\mu$ to be discrete or continuously singular, we get (1) and (2), respectively.

(5) We can mix (1)-(4) to obtain measures in $M$ with different properties on certain $\operatorname{arcs}$ of $T$.

(6) If the measures in (1)-(5) are chosen to be even, then by taking the projection of the so constructed $\nu$ onto $[-1,1]$ we get measures in the class $M(0,1)$ with the above properties.

Proof. We start the proof by recalling the well-known result $($ see $[2,6,7])$ that $\nu \in M$ if and only if

$$
\lim _{n \rightarrow \infty} \gamma_{n+1}(\nu) / \gamma_{n}(\nu)=1
$$

We also need that $\gamma_{n}(\nu)$ is an increasing function of $n$ and decreasing function of $\nu$, which are immediate consequences of the formula [2]

$$
\frac{1}{\gamma_{n}(\nu)^{2}}=\inf _{P_{n}(z)=z^{n}+\ldots} \int_{T}\left|P_{n}\right|^{2} d \nu,
$$

which in turn is an easy consequence of the orthogonality of the polynomials $p_{k}(\nu, x)$ (develop $P_{n}$ into $\left\{p_{k}(\nu, \cdot)\right\}$ ).

Without loss of generality we can assume that each $\nu_{k}^{j}$ satisfies $\left\|\nu_{k}^{j}\right\|<2 j^{-r}$, where $\|\cdot\|$ indicates total mass (throw away those which do not have this property).

We select the sequence $\left\{k_{j}\right\}$ inductively. Suppose $k_{1}, \ldots, k_{j-1}$ have already been selected and set

$$
\begin{gathered}
\mu_{0}^{j} \equiv \mu_{0}=\sum_{l=1}^{j-1} \nu_{k_{l}}^{l}+\left(\sum_{l=j+1}^{\infty} \frac{1}{l r}\right) m, \\
\mu_{k}^{j} \equiv \mu_{k}=\mu_{0}^{j}+\nu_{k}^{j}, \quad \mu_{\infty}^{j} \equiv \mu_{\infty}=\lim _{k \rightarrow \infty} \mu_{k}^{j}=\mu_{0}^{j}+\frac{1}{j^{r}} m .
\end{gathered}
$$

We claim that there exists an $N_{j}$ such that for $n \geq N_{j}$ we have

$$
1 \leq \frac{\gamma_{n+1}\left(\mu_{k}\right)}{\gamma_{n}\left(\mu_{k}\right)} \leq 1+\frac{30 r 2^{r}}{j}
$$

for all $k=0,1, \ldots, \infty$. In fact, for each $k$ the sequence $\left\{\gamma_{n}\left(\mu_{k}\right)\right\}_{n=1}^{\infty}$ monotonically tends to $\gamma^{*}\left(\mu_{k}\right)$, where

$$
\gamma^{*}\left(\mu_{k}\right)=\exp \left(\frac{1}{2} \int_{T} \log w_{k} d m\right)
$$

with $w_{k}$ equal to the density of the absolutely continuous component of $\mu_{k}$ (see [2]). We have $w_{k}=w_{0}+\sigma_{k}$, where $\sigma_{k}$ is a nonnegative measurable function on $T$ with

$$
\int_{T} \sigma_{k} d m \leq\left\|\nu_{k}^{j}\right\| \leq 2 / j^{r}
$$


Hence we deduce from the inequality between the arithmetic and geometric means that

$$
\begin{aligned}
\frac{\gamma^{*}\left(\mu_{0}\right)}{\gamma^{*}\left(\mu_{k}\right)} & =\exp \left(\frac{1}{2} \int \log \frac{w_{k}}{w_{0}} d m\right) \leq \int \frac{w_{k}}{w_{0}} d m \\
& \leq 1+10 r 2^{r} j^{r-1} \int \sigma_{k} d m<1+\frac{20 r 2^{r}}{j},
\end{aligned}
$$

where we used that, by the definition of $\mu_{0}$,

$$
w_{0} \geq \frac{1}{2 \pi}\left(\sum_{l=j+1}^{\infty} \frac{1}{l^{r}}\right) \geq \frac{1}{10 r 2^{r} j^{r-1}} .
$$

Now consider the set $S=\left\{\mu_{0}, \mu_{1}, \ldots, \mu_{\infty}\right\}$ equipped with the weak ${ }^{*}$-topology. Then $S$ is compact. If the function $f_{n}$ on $S$ is defined by

$$
f_{n}\left(\mu_{k}\right)=\min \left\{\gamma_{n}\left(\mu_{k}\right), \frac{\gamma^{*}\left(\mu_{0}\right)}{\left(1+20 r 2^{r} / j\right)}\right\},
$$

then $f_{n}$ is continuous on $S$ and the sequence $\left\{f_{n}\right\}$ monotone increasingly converges to the constant $\gamma^{*}\left(\mu_{0}\right) /\left(1+20 r 2^{r} / j\right)$ (see (4)). Hence, the convergence is uniform on $S$. Seeing that $\gamma_{n}\left(\mu_{k}\right) \leq \gamma_{n}\left(\mu_{0}\right) \leq \gamma^{*}\left(\mu_{0}\right)$, we can finally conclude that

$$
\limsup _{n \rightarrow \infty} \frac{\gamma_{n+1}\left(\mu_{k}\right)}{\gamma_{n}\left(\mu_{k}\right)} \leq 1+\frac{20 r 2^{r}}{j}
$$

uniformly in $k \in \overline{0, \infty}$, by which the existence of $N_{j}$ has been verified.

If $k_{j}, k_{j+1}, \ldots$ all tend to infinity, then for each fixed $n$ we have for the measure (3)

$$
\gamma_{n}(\nu) \rightarrow \gamma_{n}\left(\mu_{k_{j-1}}^{j-1}\right),
$$

hence we can choose numbers $K_{j}^{j}, K_{j+1}^{j}, \ldots$ so that if $k_{j} \geq K_{j}^{j}, k_{j+1} \geq$ $K_{j+1}^{j}, \ldots$ then

$$
1 \leq \frac{\gamma_{n+1}(\nu)}{\gamma_{n}(\nu)} \leq 1+\frac{40 r 2^{r}}{j-1} \quad \text { for each } N_{j-1}<n \leq N_{j} .
$$

Now let $k_{j}$ be equal to the maximum of the numbers $K_{j}^{1}, K_{j}^{2}, \ldots, K_{j}^{j}$ (which we assume to have been defined in earlier steps of the construction with the properties above). Note that this choice of $k_{j}$ does not affect the number $N_{j}$, hence we can continue our process with selecting $N_{j+1}$, then $K_{j+1}^{j+1}, K_{j+2}^{j+1}, \ldots$, then $k_{j+1}=\max \left\{K_{j+1}^{1}, \ldots, K_{j+1}^{j+1}\right\}$, and so on.

(5) shows that $\nu$ from (3) is in the class $M$.

\section{REFERENCES}

1. F. Delyon, B. Simon, and B. Souillard, From power pure point to continuous spectrum in disordered systems, Ann. Inst. H. Poincaré Phys. Théor. 42 (1985), 283-309.

2. G. Freud, Orthogonal polynomials, Akadémia Kiadó/Pergamon Press, Budapest, 1971.

3. D. S. Lubinsky, Jump distributions in $[-1,1]$ whose orthogonal polynomials have leading coefficients with a given asymptotic behavior, Proc. Amer. Math. Soc. 104 (1988), 516-524.

4. _ Singularly continuous measures in Nevai's class $M$, Proc. Amer. Math. Soc. 111 (1991), 413-420. 
5. Al. Magnus and W. Van Assche, Sieved orthogonal polynomials and discrete measures with jumps dense in an interval, Proc. Amer. Math. Soc. 106 (1989), 163-173.

6. P. Nevai, Orthogonal polynomials, Mem. Amer. Math. Soc., no. 213, Amer. Math. Soc., Providence, RI, 1979.

7. E. A. Rahmanov, On the asymptotics of the ratio of orthonormal polynomials, Math. USSR Sb. 32 (1977), 199-213.

Bolyai Institute, Szeged, Aradi V. tere 1, 6720, Hungary

Department of Mathematics, University of South Florida, Tampa, Florida 33620 